06

\title{
Асимптотическое разложение спектра поляризации двухуровневой системы в полихроматическом поле в пределе малых амплитуд
}

\author{
(C) А.Г. Антипов, С.А. Пулькин, С.В. Уварова \\ Санкт-Петербургский государственный университет, \\ 198504 Санкт-Петербург, Россия \\ e-mail: usvik2009@yandex.ru
}

Поступила в редакцию 13.09.2018 г.

В окончательной редакции 19.03.2019 г.

Принята к публикации 09.04.2019 г.

\begin{abstract}
Воздействие полихроматического поля на двухуровневую среду рассмотрено в пределе малых амплитуд. В указанном пределе получены асимптотические разложения спектров поляризации произвольного порядка малости. Полученные спектры поляризации сравнивались с результатами численного решения уравнения матрицы плотности. По сравнению с численными методами расчета асимптотическое разложение позволяет аналитически оценить вклад каждой отдельной компоненты полихроматического поля и описать межмодовое взаимодействие. Однако с увеличением интенсивности воздействующего поля вычислительная эффективность способа падает, поскольку для достижения требуемой точности приходится увеличивать порядок асимптотического разложения.
\end{abstract}

Ключевые слова: двухуровневая система, спектр поляризации, приближение малых амплитуд

DOI: $10.21883 /$ OS.2019.08.48043.246-18

\section{Введение}

Описание спектра поляризации двухуровневой атомной системы в полихроматических полях было получено в работе [1] как результат аналитического решения уравнений матрицы плотности в приближении вращающейся волны. Однако полученное решение плохо подходит для непосредственного вычисления спектра поляризации, поскольку предполагает выполнение суммирования значений, определенных на сетке точек в пространстве, размерность которого пропорциональна количеству компонент действующего поля. В результате при числе компонент поля больше десятка расчеты оказываются трудноосуществимыми.

Для того, чтобы иметь возможность вычислить спектры поляризации для большего числа компонент воздействующего поля, в работе [2] рассмотрено разложение [3] полученного в [1] решения в пределе малых амплитуд. В первом, линейном приближении в спектре поляризации возникает известный лоренцев контур [4]. Квадратичная поправка, так же как и остальные поправки четного порядка, отсутствует в асимптотическом разложении по степеням амплитуды в силу симметрии спектра воздействующего поля относительно частоты перехода. Поправка третьего порядка, также полученная в [2], позволяет описывать нелинейные эффекты и межмодовое взаимодействие. Однако поправка третьего порядка позволяет корректно описывать спектр поляризации лишь в ограниченном диапазоне малых амплитуд воздействующего поля. Чтобы расширить границы диапазона в область больших амплитуд, требуется рассматривать поправки более высокого порядка пятого, седьмого и т. п.
В настоящей работе представлено разложение для спектра поляризации (как вещественной, так и мнимой частей) двухуровневой атомной системы в полихроматических полях в пределе малых амплитуд. Это разложение есть обобщение результатов, полученных в работе [2], на случай произвольного порядка разложения по степеням амплитуд.

Спектры поляризации, вычисленные с использованием асимптотических разложений различного порядка, сравнивались с аналогичными спектрами, полученными численными методами [5,6]. Также проводилось сравнение с результатами расчетов компоненты разности заселенностей на частоте перехода, приведенными в работе [7].

\section{Разложение в пределе малых амплитуд}

При условии равенства констант продольной и поперечной релаксаций взаимодействие двухуровневой среды с полихроматическим, симметричным относительно частоты перехода $\omega_{21}$ полем

$$
E(t)=\left(E_{0}+2 \sum_{k=1}^{K} E_{k} \cos (k \Delta t)\right) \cos \omega_{21} t
$$

в приближении вращающейся волны и неподвижного атома описывается уравнением матрицы плотности

$$
\frac{d \rho}{d t}=-i[V(t), \rho]-\gamma \rho+\left(\begin{array}{cc}
\lambda_{1} & 0 \\
0 & \lambda_{2}
\end{array}\right),
$$

где $E_{k}$ - ампитуды $k$-компоненты поля, а единственный недиагональный элемент матрицы взаимодействия 
$V_{12}=V_{21}$ равен

$$
V_{12}=\frac{\Omega_{0}}{2}+\sum_{k=1}^{K} \Omega_{k} \cos (k \Delta t) .
$$

Величины $\Omega_{k}=d_{21} E_{k} / \hbar, \quad k=0, \ldots, K$ определяются амплитудами эквидистантного воздействующего поля, $d_{21}$ - дипольный момент перехода, $\Delta-$ межмодовое расстояние, $\gamma$ есть постоянная релаксации, $\lambda_{12}=\lambda_{1}-\lambda_{2}-$ разность накачек на уровни. Поляризация $P(t)$ пропорциональна недиагональному элементу матрицы плотности $\rho_{12}=\rho_{21}^{*}$ и дипольному моменту перехода.

В работе [1] приведены выражения для спектра поляризации двухуровневой атомной системы в полихроматическом поле:

$$
\begin{gathered}
\operatorname{Re}(P)_{j}=\frac{\lambda_{12} d_{21}}{4} \sum_{\mathbf{n}} \sum_{\mathbf{l}} R(\mathbf{n}, \mathbf{l})\left(\delta_{j}^{F(\mathbf{n}, \mathbf{l})}-\delta_{j}^{-F(\mathbf{n}, \mathbf{l})}\right), \\
\operatorname{Im}(P)_{j}=-\frac{\lambda_{12} d_{21}}{4} \sum_{\mathbf{n}} \sum_{\mathbf{l}} I(\mathbf{n}, \mathbf{l})\left(\delta_{j}^{F(\mathbf{n}, \mathbf{l})}+\delta_{j}^{-F(\mathbf{n}, \mathbf{l})}\right),
\end{gathered}
$$

где суммирование проводится по двум мультииндексам длиной $K: \mathbf{n}=\left\{n_{1}, n_{2}, \ldots, n_{K}\right\}$ и $\mathbf{I}=\left\{l_{1}, l_{2}, \ldots, l_{K}\right\}$. Зависящие от мультииндексов выражения представляют собой:

$$
\begin{gathered}
R(\mathbf{n}, \mathbf{l})=\frac{\gamma}{\gamma^{2}+f(\mathbf{n})^{2}} \prod_{k=1}^{K} J_{n_{k}}\left(-\frac{2 \Omega_{k}}{k \Delta}\right) J_{l_{k}}\left(\frac{2 \Omega_{k}}{k \Delta}\right), \\
I(\mathbf{n}, \mathbf{l})=\frac{f(\mathbf{n})}{\gamma^{2}+f(\mathbf{n})^{2}} \prod_{k=1}^{K} J_{n_{k}}\left(-\frac{2 \Omega_{k}}{k \Delta}\right) J_{l_{k}}\left(\frac{2 \Omega_{k}}{k \Delta}\right), \\
f(\mathbf{n})=\Omega_{0}-\sum_{k=1}^{K} k n_{k} \Delta,
\end{gathered}
$$

$\delta$ - символ Кронекера, а выражения $F$, определяющие, на какой именно частоте дает вклад соответствующий мультииндекс, равны

$$
F(\mathbf{n}, \mathbf{l})=\sum_{k=1}^{K} k\left(n_{k}+l_{k}\right) .
$$

Размерность пространства, в котором выполняется суммирование в (3), (4), т. е. суммарная длина мультииндексов $\mathbf{n}$ и $\mathbf{l}$, равна $2 K$. Поэтому вычисление спектров, используя непосредственно формулы (3), (4), становится практически неосуществимым при большом числе гармоник воздействующего поля. Чтобы решить эту проблему, в [2] предложено выполнить асимптотическое разложение [3] по степеням амплитуд компонент поля в пределе $\Omega_{k} \rightarrow 0, k=0, \ldots, K$ :

$$
\begin{aligned}
& \operatorname{Re}(P)_{j}=\frac{\lambda_{12} d_{21}}{4}\left(\operatorname{Re} P_{j}^{(1)}+\operatorname{Re} P_{j}^{(3)}+\operatorname{Re} P_{j}^{(5)}+\ldots\right), \\
& \operatorname{Im}(P)_{j}=-\frac{\lambda_{12} d_{21}}{4}\left(\operatorname{Im} P_{j}^{(1)}+\operatorname{Im} P_{j}^{(3)}+\operatorname{Im} P_{j}^{(5)}+\ldots\right),
\end{aligned}
$$

где $\operatorname{Re} P_{j}^{(N)}=\underline{O}\left(\Omega^{N}\right), \quad \operatorname{Im} P_{j}^{(N)}=\underline{O}\left(\Omega^{N}\right)-$ поправки $N$-го порядка малости в пределе малых амплитуд,
$N=1,3,5, \ldots$. Поправки четного порядка в разложениях (5) отсутствуют из-за симметрии спектра воздействующего поля (2) относительно частоты перехода. Нечетные поправки $\operatorname{Re} P_{j}^{(N)}, \operatorname{Im} P_{j}^{(N)}$ представляют собой линейные комбинации произведений неотрицательных степеней амплитуд различных компонент полихроматического поля, сумма показателей степеней равна $N$. Амплитуды поля не обязательно равны другу, но имеют одинаковый порядок малости $\Omega_{k}=\underline{O}(\Omega)$, $k=0,1, \ldots, K$. Отсюда следует, что указанная линейная комбинация произведений действительно имеет порядок $N$ по параметру малости $\Omega$.

Обобщая результаты [2] на случай асимптотического разложения произвольной длины, для поправки $N$-го порядка к мнимой части поляризации имеем

$$
\begin{aligned}
\operatorname{Im} P_{j}^{(N)} & =\sum_{n=0}^{N} \sum_{\mathbf{k}^{(n)}} \frac{\Omega_{0}^{N-n} \Omega_{k_{1}^{(n)}} \ldots \Omega_{k_{n}^{(n)}}}{n ! k_{1}^{(n)} k_{2}^{(n)} \ldots k_{n}^{(n)} \Delta^{n}} \times \\
& \times \sum_{\nu=0}^{n} \frac{(-1)^{n-v}\left(\begin{array}{l}
n \\
v
\end{array}\right) a_{v}^{(N+1-n)}}{\left(\gamma^{2}+\left(\Delta \sum_{l=1}^{v} k_{l}^{(n)}\right)^{2}\right)^{N+1-n}} \times \\
& \times\left(\delta_{j}^{k_{1}^{(n)}+\ldots+k_{n}^{(n)}}+\delta_{j}^{-k_{1}^{(n)}-\ldots-k_{n}^{(n)}}\right),
\end{aligned}
$$

где суммирование по мультииндексу $\mathbf{k}^{(n)}=\left\{k_{1}^{(n)}, \ldots, k_{n}^{(n)}\right\}$ проводится в $n$-мерном пространстве, причем каждый из индексов $k_{l}^{(n)}, l=1, \ldots, n$, изменяется в пределах от $-K$ до $K$, исключая значение 0 . Коэффициенты $a_{v}^{(l)}, v=0, \ldots, n, l=1, \ldots, N+1$, фигурирующие в (6), представляют собой полиномы степени $l$ от $\gamma$ и $\Delta$ :

$$
\begin{aligned}
& a_{v}^{(1)}=\Delta \sum_{l=1}^{\nu} k_{l}^{(n)}, \\
& a_{v}^{(2)}=\gamma^{2}-\left(a_{v}^{(1)}\right)^{2}, \\
& a_{v}^{(3)}=\left(-3 \gamma^{2}+\left(a_{v}^{(1)}\right)^{2}\right) a_{v}^{(1)}, \\
& a_{v}^{(4)}=-\gamma^{4}+6 \gamma^{2}\left(a_{v}^{(1)}\right)^{2}-\left(a_{v}^{(1)}\right)^{4}, \\
& a_{v}^{(5)}=\left(5 \gamma^{4}-10 \gamma^{2}-\left(a_{v}^{(1)}\right)^{2}+\left(a_{v}^{(1)}\right)^{4}\right)-a_{v}^{(1)} .
\end{aligned}
$$

Отметим, что $a_{0}^{(l)}=(-1)^{l / 2+1} \gamma^{l}$ для четных $l$ и обращается в 0 для нечетных $l$.

Структура формулы (6) отражает факт наличия межмодового взаимодействия. В числителе присутствует произведение амплитуд компонент воздействующего поля. Таким образом, каждое слагаемое под знаком суммирования по мультииндексу $\mathbf{k}^{(n)}$ соответствует некоторой комбинации из $N$ взаимодействующих компонент. В первом приближении $(N=1)$ межмодовое взаимодействие отсутствует, в третьем приближении описывается взаимодействие трех мод, в следующем, пятом - 
пяти и т. п. Сомножитель, стоящий при произведении амплитуд, определяет силу взаимодействия рассматриваемых компонент. Этот сомножитель представляет собой сумму из $n+1$ слагаемого со знаменателями лоренцева типа с различным характерным отношением постоянной релаксации и межмодового расстояния. Частота, на которой наблюдается результат взаимодействия, есть комбинация частот взаимодействующих компонент, что описывается последним сомножителем с двумя символами Кронекера внутри.

Схожее с (6) выражение можно получить и для $N$-й поправки в пределе малых амплитуд в асимптотическом разложении вещественной части поляризации:

$$
\begin{aligned}
& \operatorname{Re} P_{j}^{(N)}=\sum_{n=0}^{N} \sum_{\mathbf{k}^{(n)}} \frac{\Omega_{0}^{N-n} \Omega_{k_{1}^{(n)}} \ldots \Omega_{k_{n}^{(n)}} \mathcal{Y}}{n ! k_{1}^{(n)} k_{2}^{(n)} \ldots k_{n}^{(n)} \Delta^{n}} \times \\
& \times \sum_{\nu=0}^{n} \frac{(-1)^{n-\nu}\left(\begin{array}{l}
n \\
v
\end{array}\right) a_{\nu}^{(N+1-n)}}{\left(\gamma^{2}+\left(\Delta \sum_{l=1}^{v} k_{l}^{(n)}\right)^{2}\right)^{N+1-n}} \times \\
& \times\left(\delta_{j}^{k_{1}^{(n)}+\ldots+k_{n}^{(n)}}-\delta_{j}^{-k_{1}^{(n)}-\ldots-k_{n}^{(n)}}\right) .
\end{aligned}
$$

Слагаемые в асимптотическом разложении вещественной части поляризации отличаются от слагаемых в разложении мнимой части наличием множителя $\gamma$ в числителе, полиномиальные коэффициенты $b_{v}^{(l)}$, присутствующие в (7), имеют степень $l-1$, а не $l$ как в (6):

$$
\begin{aligned}
& b_{v}^{(1)}=-1, \\
& b_{v}^{(2)}=2 a_{v}^{(1)} \\
& b_{v}^{(3)}=\gamma^{2}-3\left(a_{v}^{(1)}\right)^{2}, \\
& b_{v}^{(4)}=\left(-4 \gamma^{2}+4\left(a_{v}^{(1)}\right)^{2}\right) a_{v}^{(1)}, \\
& b_{v}^{(5)}=-\gamma^{4}+10 \gamma^{2}\left(a_{v}^{(1)}\right)^{2}-5\left(a_{v}^{(1)}\right)^{4} .
\end{aligned}
$$

В первом, линейном приближении $(N=1)$ слагаемое с $n=0$ в (7) равно нулю, поскольку $b_{0}^{(2)}=0$. В слагаемом с $n=1$ внутреннее суммирование выполняется по одному лишь индексу $k \equiv k_{1}^{(1)}$ :

$$
\begin{aligned}
\operatorname{Re} P_{j}^{(1)}= & \sum_{\substack{k=-K \\
k \neq 0}}^{K} \frac{\Omega_{k} \gamma}{k \Delta}\left(-\frac{b_{0}^{(1)}}{\gamma^{2}}+\frac{b_{1}^{(1)}}{\gamma^{2}+\Delta^{2} k^{2}}\right)\left(\delta_{j}^{k}-\delta_{j}^{-k}\right)= \\
& =\sum_{\substack{k=-K \\
k \neq 0}}^{K} \frac{\Omega_{k} \Delta k}{\gamma\left(\gamma^{2}+\Delta^{2} k^{2}\right)}\left(\delta_{j}^{k}-\delta_{j}^{-k}\right) \\
& =\left\{\begin{array}{cc}
\frac{2 \Omega_{j} \Delta j}{\gamma\left(\gamma^{2}+\Delta^{2} j^{2}\right)}, & |j| \leq K, \\
0, & |j|>K .
\end{array}\right.
\end{aligned}
$$

У мнимой части поляризации в первом приближении слагаемое с $n=0$ отлично от нуля, зато обращается в нуль слагаемое $n=1, v=0$ :

$$
\begin{aligned}
\operatorname{Im} P_{j}^{(1)} & =\frac{\Omega_{0} a_{0}^{(2)}}{\gamma^{2}} 2 \delta_{j}^{0}+\sum_{\substack{k=-K \\
k \neq 0}}^{K} \frac{\Omega_{k}}{k \Delta} \frac{a_{1}^{(1)}}{\gamma^{2}+\Delta^{2} k^{2}}\left(\delta_{j}^{k}+\delta_{j}^{-k}\right) \\
& =\left\{\begin{array}{cl}
\frac{2 \Omega_{j}}{\gamma^{2}+\Delta^{2} j^{2}}, & |j| \leq K, \\
0, & |j|>K .
\end{array}\right.
\end{aligned}
$$

Таким образом, в первом приближении в спектре поляризации возникает лоренцев контур, ограниченный набором частот воздействующего поля.

В третьем приближении из (6), (7) при $N=3$ получаются формулы, представленные в [2].

\section{Результаты расчетов спектра поляризации}

На рис. 1,2 показаны результаты расчетов спектров поглощения и дисперсии, выполненные с использованием формул (6) и (7). В разложении (5) было задействовано от одного (линейное приближение по $\Omega$ ) до трех слагаемых (поправка пятого порядка по $\Omega$ ). Воздействующее полихроматическое поле состоит из 101 эквидистантных компонент $(K=50)$, амплитуды которых равны $\Omega_{0}=\Omega_{1}=\ldots=\Omega_{K}=0.1 \gamma$, расстояние между компонентами $\Delta=0.3 \gamma$. По горизонтальной оси отложены номера компонент, частота перехода соответствует частоте компоненты с индексом $j=0$.

Непрерывные кривые на рис. 1,2 показывают результаты, полученные численно: прямым решением уравнения матрицы плотности (1) методом Рунге-Кутта с последующим Фурье-преобразованием полученной временной зависимости, а также посредством разложения решения в гармоническом базисе - способе, который в литературе зачастую описывается как метод Флоке [8]. Точность численного решения весьма высока, что подтверждается отличием результатов, полученных двумя совершенно различными методами менее чем на $0.1 \%$.

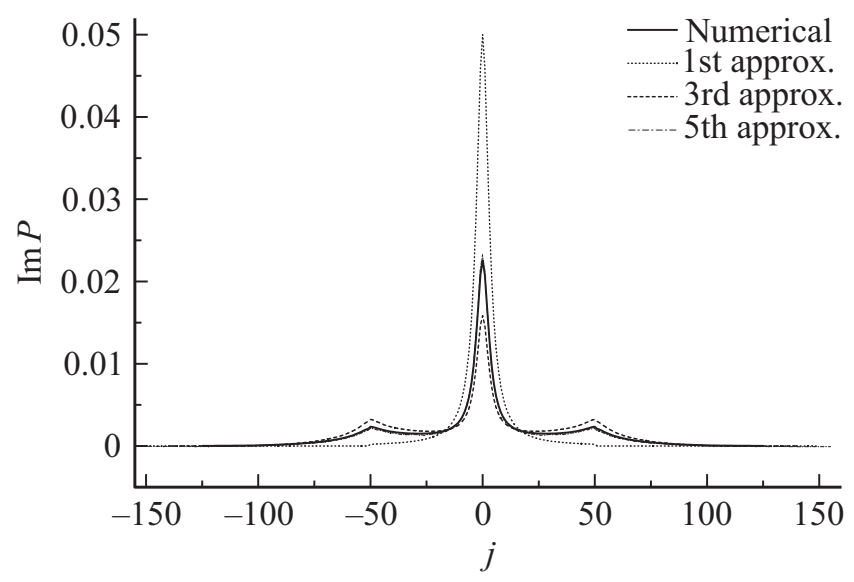

Рис. 1. Спектр мнимой части поляризации при $\Omega_{0}=\ldots=\Omega_{K}=0.1 \gamma \cdot K=50, \Delta=0.3 \gamma$. 
Как видно из рис. 1,2, при заданном значении амплитуды воздействующего поля $\Omega=0.1 \gamma$ первое, линейное приближение дает явно неудовлетворительное описание спектров поляризации: величина центрального, на частоте перехода импульса в спектре мнимой части оказывается приблизительно в 2 раза больше, чем значение, полученное численно. Побочные импульсы на частотах, определяемых границами спектра воздействующего поля, вообще отсутствуют в первом приближении.

Приближение третьего порядка по амплитуде дает качественно правильное описание спектра поляризации, однако количественные оценки недостаточно точны. Так, амплитуда центрального импульса спектра мнимой части занижена приблизительно на 30\%, амплитуда боковых - напротив, завышена на треть. Поправка пятого порядка дает гораздо более точное описание спектра при $\Omega=0.1 \gamma$ по сравнению с поправкой третьего порядка: величины центрального и боковых импульсов отличаются от результатов численного расчета на $3 \%$ и $5 \%$ соответственно.

Для проверки правильности расчетов результат сравнивался с полученными в [7] зависимостями компоненты разности заселенностей уровней на частоте перехода от амплитуды полихроматического поля. Для этого была использована связь между поляризацией и разностью заселенностей

$$
\left(\frac{d}{d t}+\gamma\right) P=i d_{21} V_{12}\left(\rho_{11}-\rho_{22}\right),
$$

вытекающая из уравнений матрицы плотности (1). Записанное в гармоническом базисе соотношение (8) дает возможность получить спектр разности заселенностей из спектра поляризации посредством обращения ленточной матрицы Тёплица [7]. Для сравнения с данными [7] нас интересует лишь компонента спектра с индексом 0 - на частоте перехода.

На рис. 3 показана зависимость компоненты разности заселенностей уровней $\left(\rho_{22}-\rho_{11}\right) / 2$ на частоте перехода от амплитуды воздействующего полихроматического

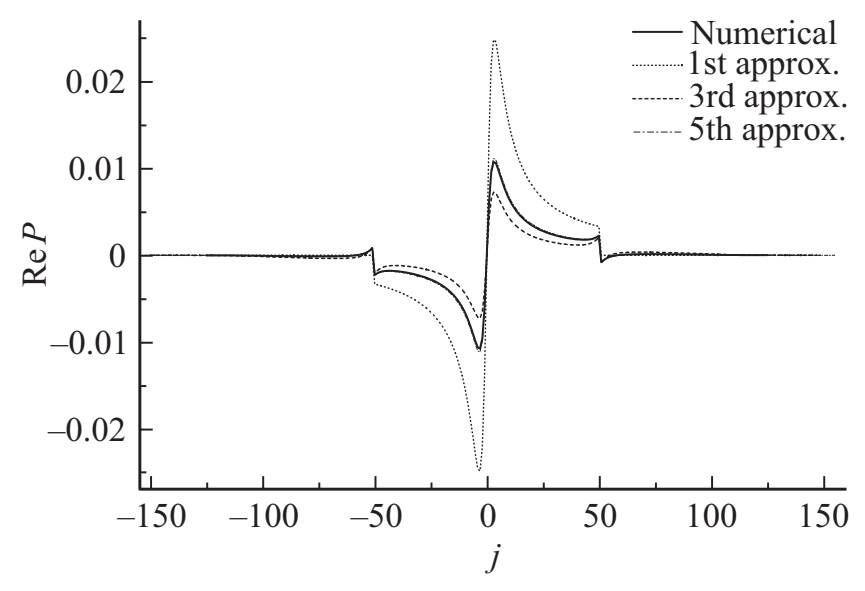

Рис. 2. Спектр вещественной части поляризации при $\Omega_{0}=\ldots=\Omega_{K}=0.1 \gamma . K=50, \Delta=0.3 \gamma$.



Рис. 3. Компонента разности заселенностей на частоте перехода в зависимости от $\Omega . \Omega=\Omega_{0}=\ldots=\Omega_{K}, K=10$, $\Delta=0.3 \gamma$.

поля. Амплитуды каждой из 21 компонент поля $(K=10)$ совпадают, расстояние между компонентами $\Delta=0.3 \gamma$. В начальном приближении, в котором принимается в расчет только одно слагаемое асимптотического ряда (5), разность заселенностей постоянна, не зависит от амплитуды действующего поля. В последующих приближениях при условии равенства амплитуд всех компонент поля, зависимость компонент спектра разности заселенностей от амплитуды представляет собой полином четной степени: второй, четвертой и т. д. С увеличением порядка приближения диапазон амплитуд, в котором асимптотическое разложение дает приемлемое описание разности заселенностей, растет. Если считать допустимым отклонение разности заселенностей уровней на частоте перехода на $1 \%$ от истинного, то первое приближение приводит к корректному значению при $\Omega \lesssim 0.03 \gamma$, третье - при $\Omega \lesssim 0.08 \gamma$, пятое - при $\Omega \lesssim 0.12 \gamma$.

\section{Выводы}

Предложен способ вычисления спектров поляризации в диапазоне малых амплитуд действующего поля. По сравнению с численными методами расчета, асимптотическое разложение позволяет аналитически оценить вклад каждой отдельной компоненты полихроматического поля и описать межмодовое взаимодействие. Однако с увеличением интенсивности воздействующего поля вычислительная эффективность способа падает, поскольку для достижения требуемой точности приходится увеличивать порядок асимптотического разложения.

Данный подход может быть обобщен на случай систем с большим числом уровней. 


\section{Финансирование работы}

Работа выполнена при частичной поддержке РФФИ (грант №18-02-01095).

\section{Конфликт интересов}

Авторы заявляют, что у них нет конфликта интересов.

\section{Список литературы}

[1] Антипов А.Г., Пулькин С.А., Сумароков А.С., Уварова С.В., Яковлева В.И. // Оптика и спектроскопия. 2015. T. 118. C. 977.

[2] Антипов А.Г,, Пулькин С.А., Уварова С.В. // Известия РАН. Серия Физическая. 2017. Т. 81. С. 1657.

[3] Copson E.T. Asymptotic Expansions. Cambridge University Press, 1965.

[4] Ficek Z., Swain S. Quantum Interference and Coherence. New York: Springer-Verlag, 2005.

[5] Antipov A.G., Kalinichev A.A., Pulkin S.A. et al. // Journal of Physics: Conference Series. 2016. V. 735. P. 012029.

[6] Антипов А.Г., Матвеева Н.И., Пулькин С.А., Уварова С.B. // Оптика и спектроскопия. 2016. Т. 121. С. 105.

[7] Ficek Z., Seke J., Soldatov A.V., Adam G. // Journal of Optics B: Quantum and Semiclassical Optics. 2000. V. 2. P. 780.

[8] Ficek Z., Seke J., Soldatov A.V., Adam G. // Physical Review A. 2003. V. 64. P. 013813.

[9] Trench W.F. // Mathematics of Computations. 1974. V. 28 P. 1089. 